\title{
Progress and prospect on imaging diagnosis of COVID-19
}

\author{
Li Fan $^{1} \cdot$ Dong $\mathrm{Li}^{2} \cdot$ Huadan Xue $^{3} \cdot$ Longjiang Zhang $^{4} \cdot$ Zaiyi Liu $^{5} \cdot$ Bing Zhang $^{6} \cdot$ Lina Zhang $^{7} \cdot$ Wenjie Yang $^{8}$. \\ Baojun $\mathrm{Xie}^{9} \cdot$ Xiaoyi Duan $^{10} \cdot$ Xiuhua Hu $^{11} \cdot$ Kailiang Cheng $^{12} \cdot$ Liqing Peng $^{13} \cdot \mathrm{Nan} \mathrm{Yu}^{14} \cdot$ Lan Song $^{3} \cdot$ Huai Chen $^{15}$. \\ Xin Sui ${ }^{3} \cdot$ Nannan Zheng ${ }^{16}$. Shiyuan Liu ${ }^{1} \cdot$ Zhengyu Jin $^{3}$
}

Received: 23 February 2020 / Revised: 23 February 2020 / Accepted: 4 March 2020 / Published online: 18 March 2020

(c) Springer Nature Singapore Pte Ltd. 2020

\begin{abstract}
COVID-19 has become a public health emergency due to its rapid transmission. The appearance of pneumonia is one of the major clues for the diagnosis, progress and therapeutic evaluation. More and more literatures about imaging manifestations and related research have been reported. In order to know about the progress and prospective on imaging of COVID-19, this review focus on interpreting the CT findings, stating the potential pathological basis, proposing the challenge of patients with underlying diseases, differentiating with other diseases and suggesting the future research and clinical directions, which would be helpful for the radiologists in the clinical practice and research.
\end{abstract}

Keywords COVID-19 $\cdot$ Imaging $\cdot$ Viral pneumonia $\cdot$ Artificial intelligence

Li Fan, Dong Li, and Huadan Xue contributed equally to the article.

\section{Shiyuan Liu}

liushiyuan@smmu.edu.cn

$\triangle$ Zhengyu Jin

jzhy_editor@163.com

1 Department of Radiology, Changzheng Hospital, Second Military Medical University, No. 415 Fengyang Road, Shanghai 200003, China

2 Department of Radiology, Tianjin Medical University General Hospital, No. 154 Anshan Road, Heping District, Tianjin 300052, China

3 Department of Radiology, Peking Union Medical College Hospital, No. 1 Shuaifuyuan, Dongcheng District, Beijing 100730, China

4 Department of Medical Imaging, Jinling Hospital, Medical School of Nanjing University, No. 305 Zhongshan Eastern Road, Xuanwu District, Nanjing 210002, China

5 Department of Radiolgy, Guangdong Provincial People's Hospital, Guangdong Academy of Medical Sciences, No. 106, Zhongshan Er Road, Guangzhou 510080, China

6 Department of Radiolgy, The Affiliated Drum Tower Hospital, Medical School of Nanjing University, No. 321 Zhongshan Road, Nanjing 210008, China

7 Department of Radiology, The First Hospital of China Medical University, No. 155 Nanjingbei Street, Heping District, Shenyang 110001, China
8 Department of Radiology, Shanghai Ruijin Hospital, No. 127 Ruijin Road, Huangpu District, Shanghai 200025, China

9 Department of Radiology, Renmin Hospital, Wuhan University, No. 238 Jiefang Road, Wuchang District, Wuhan 430060, China

10 Department of Radiology, The First Affiliated Hospital of Xi' an Jiaotong University, No. 277 Yanta West Road, Xi' an 710061, Shaanxi, China

11 Department of Radiology, Sir Run Run Shaw Hospital, Medical School, Zhejiang University, 3 East Qinchun Road, Hangzhou 310006, Zhejiang, China

12 Department of Radiology, China-Japan Union Hospital of Jilin University, No. 126 Xiantai Street, Erdao District, Changchun 130033, Jilin, China

13 Department of Radiology, West China Hospital, Sichuan University, No. 37 Guoxue Road, Wuhou District, Chengdu 610041, China

14 Department of Radiology, Affiliated Hospital of Shaanxi University of Chinese Medicine, No 2, Wei Yang West Road, Xian Yang 712000, China

15 Department of Radiology, First Affiliated Hospital of Guangzhou Medical University, No. 151 Yanjiang Road, Yuexiu District, Guangzhou 510120, China

16 Department of Radiology, Wuhan Children's Hospital (Wuhan Maternal and Child Healthcare Hospital), Tongji Medical College, Huazhong University of Science and Technologyechnology, No. 100 Hongkong Road, Jiangan District, Wuhan 430016, China 


\section{Introduction}

In early December 2019, a series of viral pneumonia cases of unknown cause emerged in Wuhan, Hubei, China [1]. This virus is a novel type of the coronavirus family, which is named 2019 novel coronavirus $(2019-\mathrm{nCoV})$ by World Health Organization (WHO) in January 7, 2020. The 2019$\mathrm{nCoV}$ will cause acute respiratory system infections, and is called coronavirus disease 19 (COVID-19). The WHO has recently declared the COVID-19 a public health emergency of international concern, because of rapid spread from Wuhan to the entire China as well as many other countries [2].

Due to its highly contagious clinical feature, rapid and reliable diagnosis of COVID-19 is very important. It can not only guide the follow-up therapies to improve outcomes, but also help to control the disease spread effectively. Chest CT is one of the key components in the diagnostic work-up for patients with suspected infection.

In this pictorial review, we focus on interpreting the CT findings, stating the potential pathological basis, proposing the challenge of patients with underlying diseases, differentiating with other diseases and suggesting the future research and clinical directions.

\section{Clinical features and diagnosis criteria}

\section{Clinical features}

The infection source is mainly the COVID-19 patients with or without symptoms. The main routes of transmission are respiratory droplets and close contact. 2019$\mathrm{nCoV}$ is highly infectious with high incidence of susceptibility crowd. According to the current epidemiological data, the mean incubation period of COVID-19 is 3 days (0-24 days) [3]. The main clinical symptoms are fever $(87.9 \%)$ and cough $(67.7 \%)$. Some mild patients show no symptoms. Serious ill patients may present with dyspnea and hypoxemia about 1 week after onset of initial symptoms, even aggravate as acute respiratory distress syndrome (ARDS), septic shock, refractory metabolic acidosis, et al.

On admission, $82.1 \%$ and $33.7 \%$ patients had lymphopenia and leukopenia, respectively. The elevated level of C reactive protein (CRP) and erythrocyte sedimentation rate (ESR) were observed. Severe cases had more prominent laboratory abnormalities (i.e., lymphopenia, leukopenia, elevated C-reactive protein levels) as compared with nonsevere cases [3]. The nucleic acid of 2019-nCoV can be detected in respiratory secretions, blood, and stool specimen, which is the evidence of definite diagnosis.

\section{Diagnosis criteria}

According to Diagnosis and Treatment Protocols of COVID-19 Infection (6th edition) (hereafter referred to as Protocols-Ver6) issued by the National Health Commission of the People's Republic of China, in February 19, 2020 [4], the diagnosis of COVID-19 should be decided by epidemiological, clinical and laboratory characteristics comprehensively.

Epidemiological histories include: (1) travel history or residence history in and around Wuhan City no earlier than 14 days before initial symptoms onset; (2) close contact history with COVID-19 patients no earlier than 14 days before initial symptoms onset; (3) contact history with patients with fever or respiratory symptoms from Wuhan City and its surrounding area no earlier than 14 days before initial symptoms onset; (4) clustering occurrence.

Clinical manifestations include: (1) fever and/or respiratory symptoms; (2) the imaging findings of COVID19 pneumonia; (3) reduced/normal total WBC count, or reduced lymphocyte count.

Suspended cases should have both one of the epidemiological histories and two of the clinical manifestations, or have three of the clinical manifestations without epidemiological history. If the nucleic acid of 2019-nCoV in samples of respiratory tract and blood was detected in suspended case by using reverse transcription polymerase chain reaction (RT-PCR) technique, then the final diagnosis should be issued.

The detection rate of nucleic acid with RT-PCR is influenced by many factors and the reaction itself is time consuming. Considering the large amounts of patients with epidemiological histories and typical clinical manifestations in Hubei Province, suspended cases with typical imaging findings would be considered as clinically diagnosed cases in Protocols-Ver5. This is only applicable in special period, and the has Protocols-Ver6 has deleted it from the diagnosis criteria. In some extent, this represents the outbreak leveling off at the release time of Protocols-Ver6.

\section{Clinical category}

According to the Protocols-Ver6 [4], COVID-19 is divided into mild type, moderate type, severe type and critical type based on its severity. (1) Mild type, clinical symptoms are mild, and no pneumonia appearance was found in imaging; (2) moderate type, with fever, respiratory symptom or others, and pneumonia appearance was found in imaging; (3) severe type, with any of the followings, respiratory distress, $\mathrm{RR} \geq 30$ times/min; oxygen saturation $\leq 93 \%$ in resting state; arterial partial pressure of oxygen $\left(\mathrm{PaO}_{2}\right) /$ concentration of 


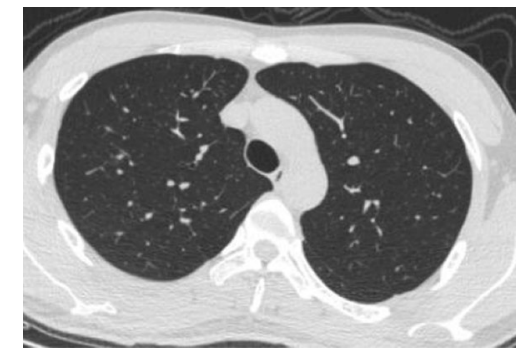

Fig. 1 Male, 30 years. A history of travel to Wuhan in the last 2 weeks, fever, normal WBC, and decreased lymphocyte count. No abnormal findings were found on CT images

oxygen $\left(\mathrm{FiO}_{2}\right) \leq 300 \mathrm{mmHg}$; fast progression on imaging findings (more than 50\%) within 24-48 h; (4) critical type, with any of the followings, respiratory failure, mechanical ventilation, shock and other organ failure resulting in the treatment in the Intensive Care Unit (ICU).

\section{Imaging manifestation of COVID-19}

\section{Imaging technique}

Chest X-ray is the most convenient and economic modality for the screening and follow up of most pulmonary diseases. However, since the early imaging manifestation of COVID19 is ground glass opacity (GGO), which could be missed by chest X-ray due to its low-resolution nature and projection overlapping. Therefore, CT is recommended for the screening of COVID-19, while chest X-ray, especially the bed side $\mathrm{X}$-ray, plays an important role in the follow-up of the severe and critical cases [5]. High resolution CT is the best choice to detect possible pulmonary opacities in COVID-19 suspicious patient. At present, low dose $\mathrm{CT}$ is not recommended for the screening of COVID-19 due to the low imaging quality and possibility of false negative GGO detection.

\section{CT findings}

COVID-19 CT findings vary with the patient's age, immunity status, disease stage, underlying diseases and drug interventions at the time of scanning [6]. In the largest cohort ( $n=1099)$ study by Guan et al. [3], 23.6\% confirmed cases with CT scanning $(n=840)$ showed no pneumonia on CT images (Fig. 1).

According to the published data [7-20], the imaging manifestations of COVID-19 are somewhat overlapping with other viral pneumonia, especially SARS, which belongs to the same viral family. According to the reported patient cohorts, the most typical chest CT findings in patients with COVID-19 include unilateral or bilateral, focal, multifocal, or diffuse areas of GGO and consolidation with subpleural, peripheral or peribronchovascular distribution [7-15]. GGO superimposed the thickening of interlobular or intralobular septa manifest as 'crazy-paving' sign. Several atypical features have been reported as well, including halo sign, reversed halo sign (the atoll sign), cavity, small pleural effusion, pneumothorax, and lymphadenopathy [16-18]. Over time and following treatment, findings of fibrosis, including architectural distortion, traction bronchiectasis and peribronchovascular thickening may be seen. The following aspects should be evaluated comprehensively to make the diagnosis in combination with the epidemiological, clinical and laboratory characteristics.

1. Distribution pattern

The distribution of the pulmonary disease depends on the pathogenesis. The most frequent distribution is subpleural or peripheral area $(86 \%, 44 / 51)$ [18] (Figs. 2, 3 ). Moreover, the lower lobe (53\%) and posterior lung $(89 \%)$ is more prone to be involved [19]. Occasionally, inner zone of lung can be affected (Fig. 4). Peri-bronchovascular bundle thickening can be found (Fig. 5).

2. Quantity and range

Some cases show uni-focal lesion as the initial CT finding, then multifocal with the disease progression; while some show multifocal lesions at onset. $71 \%$ $(15 / 21)$ patients had involvement of at least two lobes [20]; 63\% (32/51) patients involved four to five lobes [19] (Fig. 6).

3. Density

The density of lesion is usually heterogeneous. GGO is the most common imaging feature, presenting in about $50 \%(550 / 1099)$ cases. Consolidation $(37.2 \%, 409 / 1099)$ [3], GGO with consolidation $(59 \%, 30 / 51)$ [19], and fibrosis [12] can occur in severe type cases (Fig. 7). The first pathological findings of COVID-19 by postmortem biopsy showed the bilateral diffuse alveolar damage with cellular fibromyxoid exudate, which is the basis of GGO and consolidation [21].

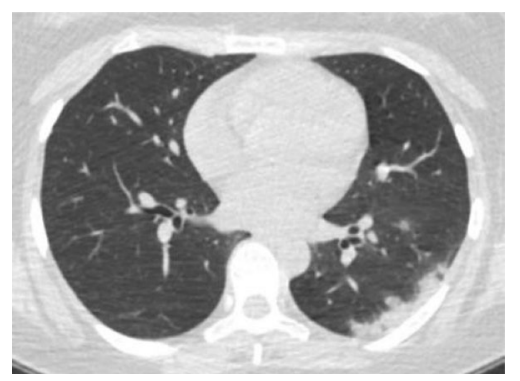

Fig. 2 Female, 34 years. Close contact with confirmed case, fever, cough, short breath, decreased WBC and lymphocyte count. CT shows subpleural consolidation and ill-defined nodule in the posterior basal segment of left lower lobe 
Fig. 3 Male, 51 years. A history of travel to Wuhan in the last 2 weeks, fever, cough, decreased WBC and lymphocyte count, elevated CRP. CT shows bilateral, multiple and subpleural GGO and consolidation
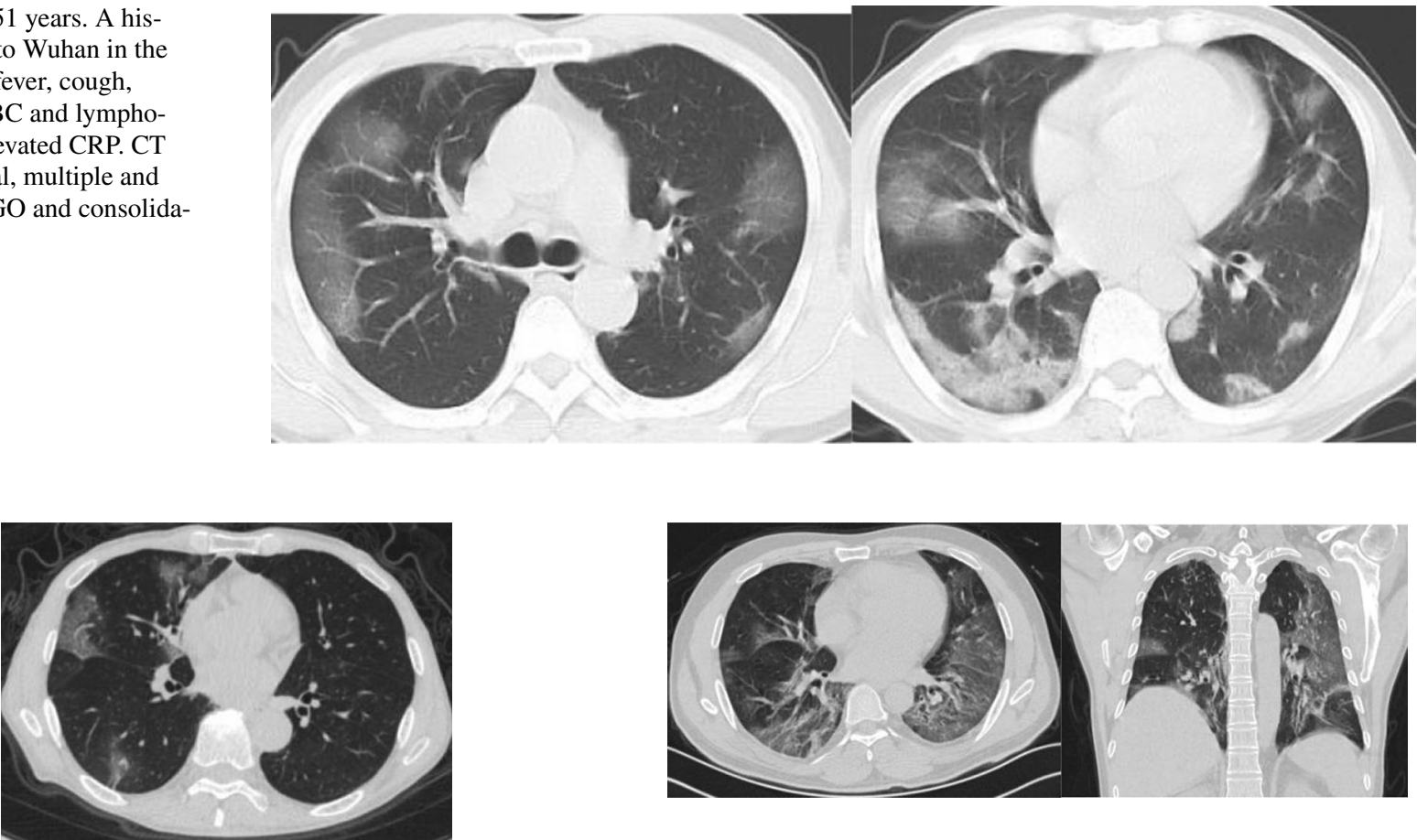

Fig. 4 Male, 66 years. A history of travel to Wuhan in the last 2 weeks, fever, cough, decreased WBC and lymphocyte count, elevated CRP and LDH. CT shows multiple GGO with subpleural and peribronchovascular distribution, involvement of the inner zone of right middle lobe

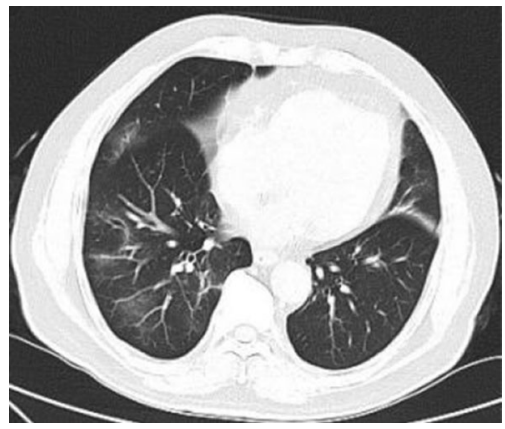

Fig. 5 Male, 68 years. Cluster transmission, fever, cough, normal WBC, decreased lymphocyte count, and elevated CRP. CT shows multiple GGO with peribronchovascular distribution and sparing the pleural surface

\section{Shape and interface}

The shape is related to the distribution pattern. Common shapes include patchy, cone-shape or triangle, subsegmental, and segmental. Sometimes, round or centrilobular nodule with halo sign [17] or reversed halo sign [18] also can be seen. The interface between lesion and the normal pulmonary is often ill-defined. Occasionally, the mosaic perfusion may be present (Figs. 8, 9, 10, 11,

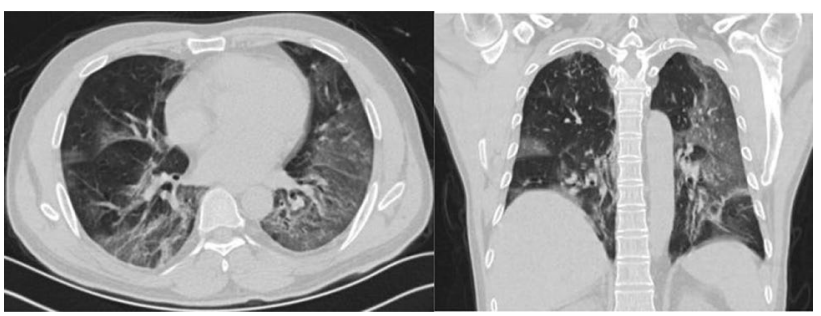

Fig. 6 Male, 50 years. A history of travel to Wuhan in the last 2 weeks, fever, cough, short breath, hypoxemia, normal WBC, decreased lymphocyte count, and elevated CRP and LDH. CT shows multiple GGO involvement of five lobes

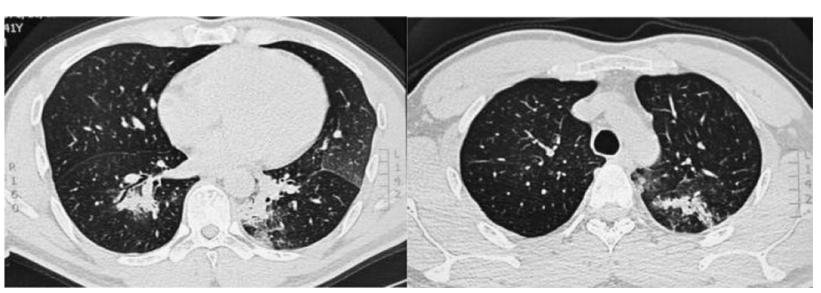

Fig. 7 Male, 41 years. A history of travel to Wuhan in the last 2 weeks, fever, cough, decreased WBC lymphocyte count, elevated CRP. CT shows multiple consolidation, GGO, and AB sign

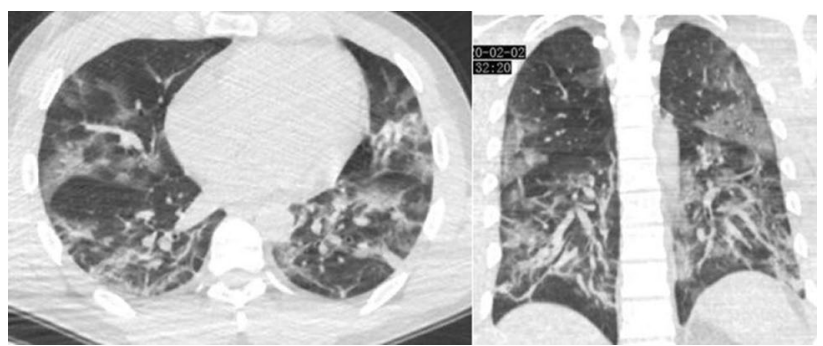

Fig. 8 Male, 40 years. A history of travel to Wuhan in the last 2 weeks, no fever, no cough, normal WBC, decreased lymphocyte count, elevated CRP and ESR. CT shows multiple patchy, ill-defined consolidation, GGO, thickening of intralobular septa and fibrosis. AB sign and mosaic perfusion was seen 


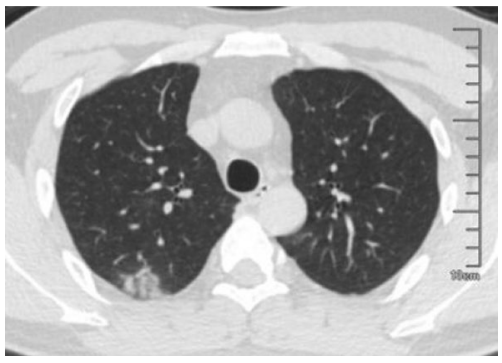

Fig. 9 Male, 29 years. A history of travel to Wuhan in the last 2 weeks, fever, sore throat, normal WBC and lymphocyte count. CT shows multiple centrilobular nodules, thickening of the adjacent small vessels, and cone-shape in the apical segment of right upper lobe

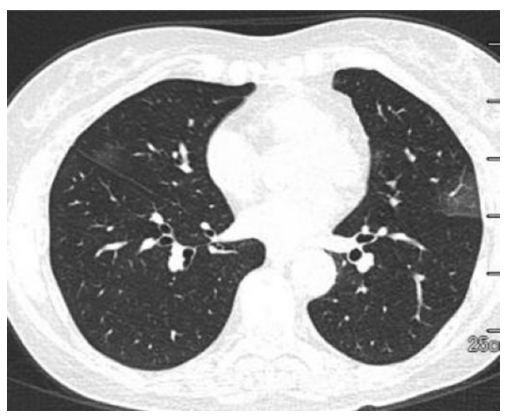

Fig. 10 Female, 58 years. Close contact with confirmed case, fever, cough, nasal congestion, and runny nose. CT shows multiple subpleural GGO with thickening of small vessel, and cone-shape in the left upper lobe

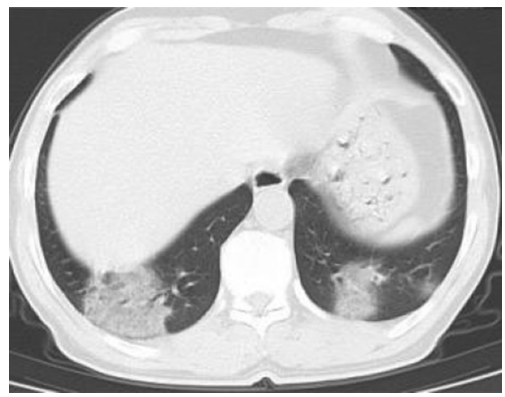

Fig. 11 Male, 51 years. A history of travel to Wuhan in the last 2 weeks, fever, cough, decreased WBC and lymphocyte count, elevated CRP. CT shows bilateral, round GGO with $A B$ sign

$12,13)$. Halo sign is defined as a circle of GGO surrounding a soft tissue density lung nodule, may be seen in some patients with a solitary pulmonary nodule. The histologic nature of halo varies with the disease, such as the hemorrhage, and lipid spread of tumor. The halo sign in patients with COVID-19 may be caused by the alveolar edema and hemorrhage. While reversed-halo

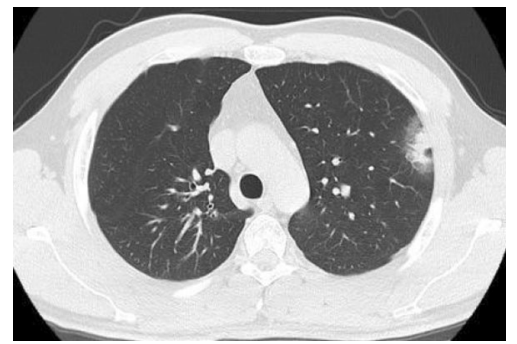

Fig. 12 Male, 25 years. A history of travel to Vietnam, fever, cough, normal WBC, decreased lymphocyte count and elevated CRP. CT shows consolidation surrounded by the GGO (halo sign) in the left upper lobe

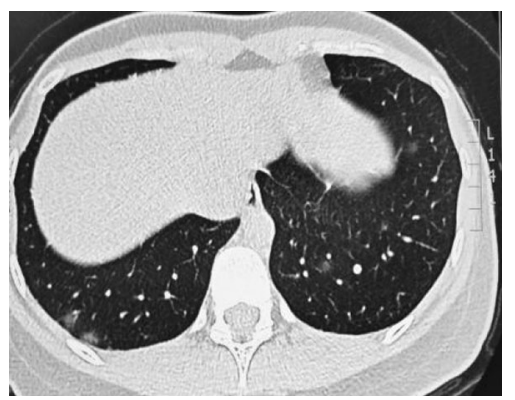

Fig. 13 Female, 21 years. A history of travel to Wuhan in the last 2 weeks, fever, cough, nasal congestion, runny nose and diarrhea, decreased WBC and lymphocyte count, elevated CRP. CT shows multiple small patchy GGO with ill-defined margin

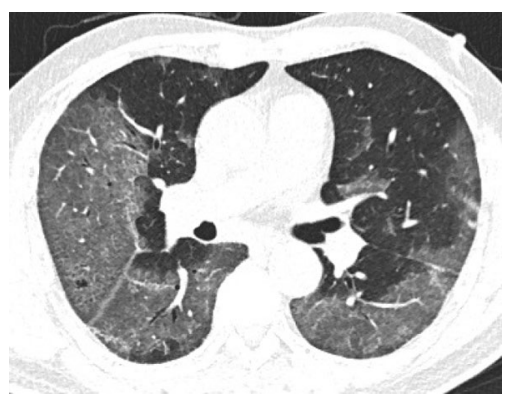

Fig. 14 Male, 65 years. No epidemiological history, fever, cough, hypoxemia, normal WBC, decreased lymphocyte count, elevated ESR and LDH. CT shows subpleural GGO with AB sign, thickening of intralobular septa (crazy-paving sign) and thickening of small vessels

sign may be related to the organization and resolution of alveolar exudates.

5. Internal feature in the lesion

The typical and most common internal features are 'crazy-paving' sign (GGO superimposed the thickening of interlobular or intralobular septa), thickening of small vessels with natural course, and air bronchogram (AB) $(80 \%, 41 / 51)$. Sometimes, bronchovascular bundle 


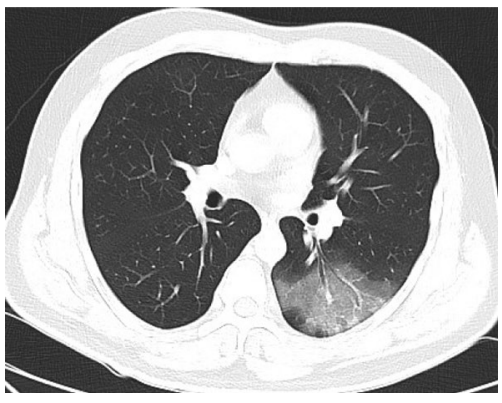

Fig. 15 Male, 42 years. A history of travel to Wuhan in the last 2 weeks, fever, cough, nasal congestion, and runny nose, normal WBC, decreased lymphocyte count, elevated CRP. CT shows GGO with $\mathrm{AB}$ sign and thickening of small vessels

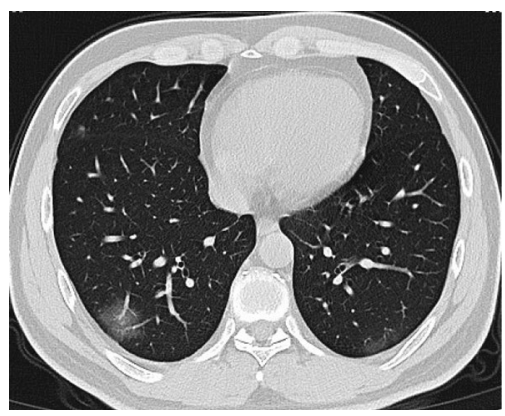

Fig. 16 Male, 33 years. A history of close contact with the fever person from Wuhan, fever, mild chest tightness, normal WBC, decreased lymphocyte count, elevated CRP, ESR and LDH. CT shows GGO and thickening of small vessels with natural course in the right lower lobe

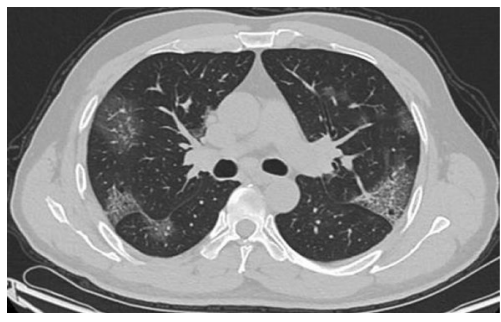

Fig. 17 Male, 49 years. Close contact with the confirmed case, fever, cough, hypoxemia, normal WBC, decreased lymphocyte count, elevated CRP, ESR and LDH. CT shows multiple GGO with crazypaving sign, thickening of small vessels and shifted bilateral oblique fissure

is thickened within the lesion. Cavity can occur in occasion [16] (Figs. 14, 15, 16).

6. Adjacent structures changes

The common changes of adjacent structures include the thickening and/or shifting of pleural (Fig. 17). The subpleural region may be spared (Fig. 18) or not [22], speculating related to the amount of alveolar exudates

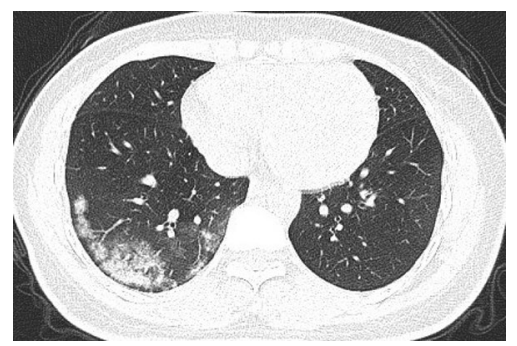

Fig. 18 Female, 38 years. A history of close contact with the fever person from Wuhan, cough, mild chest tightness, normal WBC, decreased lymphocyte count, elevated CRP and LDH. CT shows subpleural consolidation sparing the pleural surface in the right lower lobe

and the distance to the pleural. Pleural effusion, pericardial effusion and lymphadenopathy are rare.

\section{CT staging system}

CT staging system is important to evaluate the disease progress and evolution. In general, CT staging system is based on the pathological change, the combination of CT findings and pathological features could provide more accurate information. The first CT staging system was reported by Shi $\mathrm{H}$, in which COVID-19 CT findings is classified into early stage, progressive stage and severe stage [7]. Then, taking clinical manifestation and pathologic changes into account, Jin proposed a five-stages system which include ultra-early stage, early stage, rapid progression stage, consolidation stage and dissipation stage [6]. The first CT staging system is based on the imaging findings alone; while the second one is mainly based on the days of symptom onset. In practice, the imaging findings and clinical symptoms may not match. Therefore, the most optimized CT staging system should be based on the evolution of pathology.

\section{Quantitative CT and AI}

CT findings of COVID-19 depend heavily on its disease severity. It has been reported that CT quantitation of pneumonia in the severe and critical type was significantly higher than that of moderate type [8]. The ratio of pneumonia volume to the entire lungs can be used as a quantitative parameter of disease severity evaluation. The quantitative evaluation of pneumonia volume has been defined as an important indicator for management of the severe type patients. Several AI assistant diagnosis systems have already being developed and are being tested in hospitals currently, focusing on the intelligent scanning, diagnosis and severity evaluation, in order to minimize the contact transmission, improve the diagnostic accuracy and guide the treatment. 


\section{Imaging outcome and dynamic change during follow up}

The evolution process of COVID-19 on CT image is not quite clear yet. Feng et al. [23] reviewed 21 patients recovering from COVID-19 (without severe respiratory distress during the disease course). The mean interval between baseline and follow-up CT scans was $4 \pm 1$ days, and the mean hospitalization duration was $17 \pm 4$ days. The majority of cases with COVID-19 were controlled with stable and improving conditions after effective personalized treatment. The peak severity was at approximately 10 days and the improvement happened at approximately 14 days after initial onset of symptoms. The follow-up CT showed that the lesions in lungs were decreased in both number and size. The density of lesions was reduced as well $[12,24]$. The CT findings of clinically cured cases showed full recovery or only small amounts of residual stripes (Fig. 19). For some cases, the GGO and consolidation in lungs became some thick fibrotic stripes in short term, which need follow-up studies to explore its final outcome.

Some cases with COVID-19 failed to receive effective therapy will reach their deterioration and the changes will be rather rapid. The lesions on CT will become more diffuse distributed in a truly short-term [25].

\section{Imaging presentation of COVID-19 in Children}

Most children cases with COVID-19 are characterized as family cluster disease. The symptoms in children with COVID-19 are mild and the prognosis is very good. The nucleic acid of 2019-nCoV should be examined with anal swab, which is more sensitive than throat swab in children.

Chest X-ray may show no abnormality, therefore it is not recommended as a screening tool in children. CT findings of COVID-19 in children were variable and non-specific.

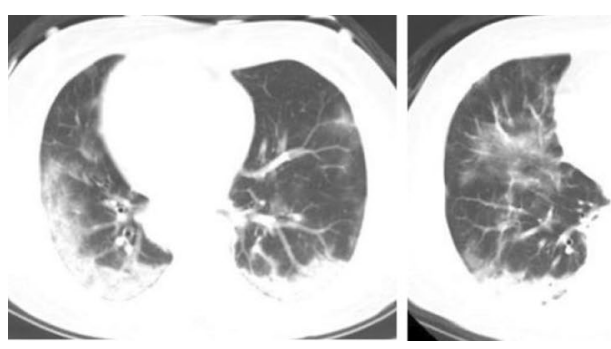

Fig. 19 Female, 62 years. From Wuhan, fever for 5 days, normal WBC and decreased lymphocyte count, elevated CRP and PCT. The CT obtained at first day after admission showed bilateral, multiple and subpleural GGO and consolidation. The follow-up CT 3 days

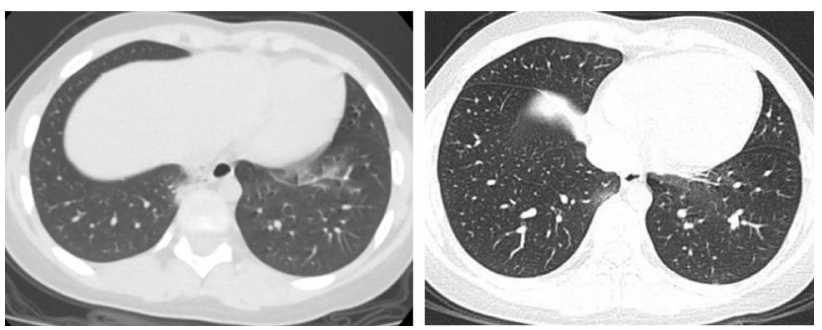

Fig. 20 Female, 8 years. Clustering occurrence, fever, pharyngalgia, normal WBC and decreased lymphocyte count, elevated CRP. CT showed bilateral, multiple and subpleural GGO and consolidation. The follow-up CT 5 days later showed the lesions were smaller in size and lighter in density

The GGO and consolidation were also the most common findings in bilateral lungs, while the lesions in children were less diffused than adults [25]. One relative specific CT characteristics in children was the presentation mimic bronchopneumonia. Pleural effusion and lymphadenopathy were also not observed (Fig. 20).

For suspicious children cases, CT findings can help to setup treatment plan in time, as well as patient isolation to avoid further contamination. While, the final diagnosis should be confirmed by laboratory examination (PCR).

\section{COVID-19 complicated with underlying disease}

Common underlying diseases include chronic obstructive pulmonary disease, diabetes, hypertension, coronary heart disease, cerebrovascular diseases, hepatitis B infection, cancer, chronic renal diseases, immunodeficiency, hematopathy, et al. The largest cohort study showed that $25.2 \%(255 / 1099)$ patients coexisted at least one underlying disease, and the ratio of underlying cases in severe group was significantly higher than that in non-severe group $(38.2 \%$ vs. $22.5 \%$, $P<0.001$ ) [3]. Patients with underlying disease may result in more severe COVID-19 [1], there is yet no literatures

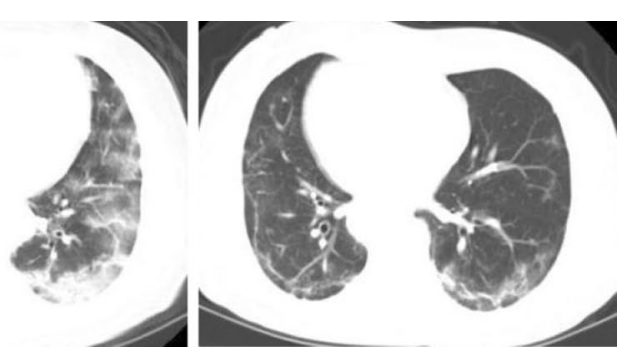

later showed the consolidation were smaller in size and the GGO became diffused. The follow-up CT 16 days later showed consolidation and GGO were resolved instead of fibrous strips 
about the COVID-19 imaging manifestation in patients with underlying pulmonary diseases. Patients with potential disease, especially for immunodeficiency, are more prone for the infection, especially the viral infection $[26,27]$. In clinical practice, the differential diagnosis should include the disease associated with the underlying disease. The imaging features of patients with underlying disease should be accumulated and investigated.

\section{Differential diagnosis}

The differential diagnosis between COVID-19 and other pulmonary infection and/or non-infectious disease is very important but also very difficult due to its overlapping imaging features. The epidemiological history, clinical symptoms, laboratory and imaging manifestations should be considered comprehensively before diagnosis is reached. But the confirmed diagnosis should be based on the etiology eventually. Although definitive diagnosis cannot be made on the basis of imaging features alone, the imaging patterns may help to improve the accuracy of diagnosis in this disease. According to the imaging findings of COVID-19 summarized by the current confirmed cases, it should be differentiated with other viral pneumonia, bacterial pneumonia, mycoplasmal pneumonia as well as many non-infectious diseases.

\section{Other viral pneumonia}

Imaging patterns can help to identify the viral pathogens. Viruses in the same viral family share a similar pathogenesis of pneumonia, and the imaging patterns have distinguishable characteristics for the entire viral family. The spectrum of CT findings can be classified into five categories: (a) parenchymal attenuation disturbances; (b) ground glass opacity and consolidation; (c) nodules, micronodules, and tree-in-bud opacities; (d) interlobular septal thickening; and (e) bronchial and/or bronchiolar wall thickening [28]. Some selected viral pneumonias including influenza, respiratory syncytial virus (RSV), adenovirus, and coronavirus are discussed below.

Influenza viruses are members of the Orthomyxoviridae family. They can be divided into three groups (A, B, and $\mathrm{C})$ according to the internal membrane and nucleoprotein antigens [29]. Influenza (A) are more common in infancy, while often mild and restricted to the upper respiratory tract in immunocompetent adults. In recent years, influenza virus has been reported as a major cause of pneumonia in immunocompromised patients. The pathogenesis is the destruction of airway epithelial barrier, resulting in necrotizing bronchitis and diffuse alveolar damage [30]. The predominant CT findings are unilateral or bilateral ground-glass opacities with or without associated focal or multifocal areas of consolidation, with the predominant peribronchovascular and subpleural distribution.

$R S V$ is the most frequent viral cause of lower respiratory tract infection in infants [31]. The pathogenesis is the destruction of bronchial and alveolar epithelium with small airway obstruction, and the typical CT findings are multiple centrilobular nodules and bronchial wall thickening [29].

Adenovirus accounts for 5-10\% of acute respiratory infections in infants and children [32]. Meanwhile, it is also a common cause in immunocompromised individuals, especially stem cell and solid organ transplant recipients. The pathogenesis is the bronchiolar and alveolar damage. The radiographic manifestations are multifocal consolidation in a lobular or segmental distribution and/or bilateral groundglass opacities with a random distribution [33]. Lobar collapse, especially of the right upper lobe is common in children [28].

Coronaviruses include severe acute respiratory syndrome coronavirus (SARS) and Middle East respiratory syndrome coronavirus (MERS), which were outbroken in 2003 and 2012 respectively [34, 35]. COVID-19 also belongs to this viral family. Therefore, their pathogenesis and imaging presentations were quite similar. SARS manifested as unilateral or bilateral ground-glass opacities, consolidation, thickening of the intralobular interstitium or interlobular septa and a crazy-paving appearance due to diffuse alveolar damage. Cavitation, lymphadenopathy, and pleural effusion are rare [36]. MERS pneumonia appears as subpleural and basilar airspace lesions, with extensive GGO. Consolidation is relatively rare in MERS, while more common in COVID19. Crazing-paving appearance is usually with the distortion of pulmonary architecture in SARS in comparison with COVID-19. Pleural effusion and pneumothorax are more common in MERS [37].

\section{Bacterial pneumonia}

Streptococcus pneumonia is the most common cause of pneumonia associated with Gram-positive cocci [27]. Typical clinical symptoms consist of abrupt onset of high fever, rusty brown sputum, shaking chills and chest pain. CT images show the unilateral or bilateral, lobar or segment consolidation, extending to pleural surfaces. And the air bronchogram and pleural effusion is very common.

\section{Mycoplasmal pneumonia}

Mycoplasmal pneumonia is a common cause of communityacquired pneumonia, and mostly occurs in young patients. 
The most common pathogenesis is the direct cytotoxicity and damage incurred from the host inflammatory response [38]. The clinical symptoms are similar with those of viral infection, such as dry cough, fever, and malaise. The CT images manifest as patchy, segmental, and lobular consolidation or ground glass opacity, and sometimes showing the thickening of perihilar interstitium, which is different from the COVID-19.

\section{Non-infectious diseases}

Until now, GGO is one of the major CT findings of COVID19. Some non-infectious diseases, such as interstitial pulmonary edema, hypersensitivity pneumonitis, and alveolar proteinosis, manifest as GGO. Patients with interstitial pulmonary edema usually have the definite clinical history, and the corresponding CT images show the smooth interlobular septal thickening, thickening of the peribronchovascular interstitium and GGO. Hypersensitivity pneumonitis is an allergic lung disease caused by exposure to the antigen. The typical CT imaging show patchy GGO and small ill-defined centrilobular GGN. The clinical symptoms, known exposure to antigen and GGO on CT can make the clinical diagnosis. The typical CT findings of alveolar proteinosis include the bilateral areas of GGO, smooth interlobular septal thickening, consolidation, and geographic distribution [38].

\section{Conclusion}

CT imaging findings cannot be used as gold-standard of COVID-19 diagnosis due to its overlapping and non-specific features with many other pulmonary diseases. However, it still plays a great role in detecting pneumonia existence, assessing disease progression as well as monitoring treatment response. COVID-19 imaging manifestation in patients with underlying diseases has not been investigated comprehensively. Moreover, multicenter study including the epidemiological, clinical and laboratory characteristics, and imaging should be performed to improve the diagnosis, guide the treatment and accelerate the implementation of isolation measures, then control the transmission.

Acknowledgements We appreciate the contribution of Drs. Jiong Ni (Tongji Hospital, Tongji University School of Medicine), Weiwei Chen (Tongji hospital, Tongji Medical college, Huazhong University of Science and Technology), Jianghong Chen (Beijing Friendship Hospital Capital Medical University), Junping Zhen (The Second Hospital of Shanxi Medical University), Miao Chang (The First Affiliated Hospital of China Medical University), Xiaodan Ye (Shanghai Chest Hospital, Shanghai Jiaotong University), and Zhigang Chu (The First Affiliated Hospital of Chongqing Medical University), who greatly facilitate the collection of patient's CT data.

\section{Compliance with ethical standards}

Conflict of interest The authors declare that they have no competing interests.

\section{References}

1. Huang C, Wang Y, Li X, et al. Clinical features of patients infected with 2019 novel coronavirus in Wuhan, China. Lancet. 2020;395(10223):497-506. https://doi.org/10.1016/S0140 $-6736(20) 30183-5$.

2. WHO main website. https://www.who.int. Accessed 5 Feb 2020 https://doi.org/10.1073/pnas.1502972112.

3. Guan W-J, Ni Z-Y, Hu Y, et al. Clinical characteristics of 2019 novel coronavirus infection in China. medRxiv. 2020. https://doi. org/10.1101/2020.02.06.20020974.

4. Diagnosis and Treatment Protocols of COVID-19 Infection (Trial Version 6). The National Health Commission of the People's Republic of China.

5. Radiology CSo. Radiological diagnosis in novel coronavirus pneumonia: expert recommendation from the Chinese Society of Radiology (version 1). Chin J Radiol. 2020;54:E001-E. https:// doi.org/10.3760/cma.j.issn.1005-1201.2020.0001.

6. Jin Y-H, Cai L, Cheng Z-S, et al. A rapid advice guideline for the diagnosis and treatment of 2019 novel coronavirus $(2019-\mathrm{nCoV})$ infected pneumonia (standard version). Mil Med Res. 2020;7(1):4. https://doi.org/10.1186/s40779-020-0233-6.

7. Shi H, Han X, Fan Y, et al. Radiologic features of patients with 2019-nCoV infection. J Clin Radiol. 2020. https://doi. org/10.13437/j.cnki.jcr.20200206.002.

8. Huang LHR, Yu P, et al. The correlation between clinical characteristics and CT findings in different clinical stage in COVID-19. Chin J Radiol. 2020;54(00):E003. https://doi.org/10.3760/cma.j. issn.1005-1201.2020.0003 (in Chinese).

9. Liu P, Tan X-z. Novel coronavirus (2019-nCoV) pneumonia. Radiology. 2019;2020:200257. https://doi.org/10.1148/radiol.20202 00257.

10. Kanne JP. Chest CT findings in 2019 novel coronavirus (2019$\mathrm{nCoV}$ ) infections from Wuhan, China: key points for the radiologist. Radiology. 2020;2020:200241. https://doi.org/10.1148/radio 1.2020200241.

11. Xie X, Zhong Z, Zhao W, et al. Chest CT for typical 2019-nCoV pneumonia: relationship to negative RT-PCR testing. Radiology. 2020;2020:200343. https://doi.org/10.1148/radiol.2020200343.

12. Shi H, Han X, Zheng C. Evolution of CT manifestations in a patient recovered from 2019 novel coronavirus $(2019-\mathrm{nCoV})$ pneumonia in Wuhan, China. Radiology. 2020;2020:200269. https ://doi.org/10.1148/radiol.2020200269.

13. Qian L, Yu J, Shi H. Severe acute respiratory disease in a Huanan seafood market worker: images of an early casualty. Radiol Cardiothorac Imaging. 2020;2(1):e200033. https://doi.org/10.1148/ ryct. 2020200033.

14. Liu T, Huang P, Liu H, et al. Spectrum of chest CT findings in a familial cluster of COVID-19 infection. Radiol Cardiothorac Imaging. 2020;2(1):e200025. https://doi.org/10.1148/ryct.20202 00025 .

15. Huang P, Liu T, Huang L, et al. Use of chest $\mathrm{CT}$ in combination with negative RT-PCR assay for the 2019 novel coronavirus but high clinical suspicion. Radiology. 2020;2020:200330. https://doi. org/10.1148/radiol.2020200330. 
16. Kong W, Agarwal PP. Chest imaging appearance of COVID-19 infection. Radiol Cardiothorac Imaging. 2020;2(1):e200028. https ://doi.org/10.1148/ryct.2020200028.

17. Li X, Zeng X, Liu B, et al. COVID-19 infection presenting with CT Halo sign. Radiol Cardiothorac Imaging. 2020;2(1):e200026. https://doi.org/10.1148/ryct.2020200026.

18. Fang $\mathrm{Y}$, Zhang $\mathrm{H}, \mathrm{Xu} \mathrm{Y}$, et al. CT manifestations of two cases of 2019 novel coronavirus (2019-nCoV) pneumonia. Radiology. 2020;2020:200280. https://doi.org/10.1148/radiol.2020200280.

19. Song F, Shi N, Shan F, et al. Emerging coronavirus 2019nCoV pneumonia. Radiology. 2020;2020:200274. https://doi. org/10.1148/radiol.2020200274.

20. Chung M, Bernheim A, Mei X, et al. CT imaging features of 2019 novel coronavirus (2019-nCoV). Radiology. 2020;2020:200230. https://doi.org/10.1148/radiol.2020200230.

21. Xu Z, Shi L, Wang Y, et al. Pathological findings of COVID-19 associated with acute respiratory distress syndrome. Lancet Respir Med. 2020. https://doi.org/10.1016/S2213-2600(20)30076-X.

22. Lei J, Li J, Li X, et al. CT imaging of the 2019 novel coronavirus (2019-nCoV) pneumonia. Radiology. 2020;2020:200236. https:// doi.org/10.1148/radiol.2020200236.

23. Pan F, Ye T, Sun P, et al. Time course of lung changes on chest CT during recovery from 2019 novel coronavirus (COVID-19) pneumonia. Radiology. 2020;2020:200370. https://doi.org/10.1148/ radiol.2020200370.

24. Duan Y-N, Qin J. Pre- and posttreatment chest CT findings: 2019 novel coronavirus (2019-nCoV) pneumonia. Radiology. 2020;2020:200323. https://doi.org/10.1148/radiol.2020200323.

25. Jiang N, Zheng C, Fan Y, et al. CT appearance of new coronavirus pneumonia in subclinical period and short-term changes. Chin J Radiol. 2020;54:E008-E. https://doi.org/10.3760/cma.j.i ssn.1005-1201.2020.0008 (in Chinese).

26. Choi MH, Jung JI, Chung WD, et al. Acute pulmonary complications in patients with hematologic malignancies. RadioGraphics. 2014;34(6):1755-68. https://doi.org/10.1148/rg.346130107.

27. Garg M, Prabhakar N, Gulati A, et al. Spectrum of imaging findings in pulmonary infections. Part 1: bacterial and viral. Pol J Radiol. 2019;84:e205-e13. https://doi.org/10.5114/pjr.2019.85812.

28. Franquet T. Imaging of pulmonary viral pneumonia. Radiology. 2011;260(1):18-39. https://doi.org/10.1148/radiol.11092149.
29. Koo HJ, Lim S, Choe J, et al. Radiographic and CT features of viral pneumonia. RadioGraphics. 2018;38(3):719-39. https://doi. org/10.1148/rg.2018170048.

30. Taubenberger JK, Morens DM. The pathology of influenza virus infections. Annu Rev Pathol. 2008;3(1):499-522. https://doi. org/10.1146/annurev.pathmechdis.3.121806.154316.

31. Sinaniotis CA. Viral pneumoniae in children: incidence and aetiology. Paediatr Respir Rev. 2004;5:S197-200. https://doi. org/10.1016/S1526-0542(04)90037-1.

32. Buckwalter SP, Teo R, Espy MJ, et al. Real-time qualitative PCR for 57 human adenovirus types from multiple specimen sources. J Clin Microbiol. 2012;50(3):766-71. https://doi.org/10.1128/ JCM.05629-11.

33. Chong S, Lee KS, Kim TS, et al. Adenovirus pneumonia in adults: radiographic and high-resolution CT findings in five patients. Am J Roentgenol. 2006;186(5):1288-93. https://doi.org/10.2214/ AJR.05.0128.

34. Cleri DJ, Ricketti AJ, Vernaleo JR. Severe acute respiratory syndrome (SARS). Infect Dis Clin N Am. 2010;24(1):175-202. https ://doi.org/10.1016/j.idc.2009.10.005.

35. Hui DS, Memish ZA, Zumla A. Severe acute respiratory syndrome vs the Middle East respiratory syndrome. Curr Opin Pulm Med. 2014;20(3):233-41. https://doi.org/10.1097/mcp.0000000000 000046.

36. Wong KT, Antonio GE, Hui DSC, et al. Severe acute respiratory syndrome: radiographic appearances and pattern of progression in 138 patients. Radiology. 2003;228(2):401-6. https://doi. org/10.1148/radiol.2282030593.

37. Das KM, Lee EY, Jawder SEA, et al. Acute Middle East respiratory syndrome coronavirus: temporal lung changes observed on the chest radiographs of 55 patients. Am J Roentgenol. 2015;205(3):W267-74. https://doi.org/10.2214/AJR.15.14445.

38. Webb WR, Higgins CB. Thoracic imaging: pulmonary and cardiovascular radiology, 3rd edn. Wolters Kluwer, 2017.

Publisher's Note Springer Nature remains neutral with regard to jurisdictional claims in published maps and institutional affiliations. 